\title{
The effect of surgical suture material on osteoclast generation and implant-loosening
}

\author{
Ulrike Dapunt ${ }^{\bowtie}$, Birgit Prior², Jan Philippe Kretzer³, Gertrud Maria Hänsch4, Matthias Martin Gaida ${ }^{5}$ \\ 1. Center for Orthopaedics, Trauma Surgery and Spinal Cord Injury, Heidelberg University Hospital, Schlierbacher Landstrasse, Heidelberg, Germany. \\ 2. Department of Anesthesiology, Heidelberg University Hospital, Heidelberg. \\ 3. Laboratory of Biomechanics and Implant Research, Center for Orthopaedics, Trauma Surgery and Spinal Cord Injury, Heidelberg University Hospital, \\ Heidelberg, Germany. \\ 4. Institute of Immunology, Heidelberg University. Heidelberg, Germany. \\ 5. Institute of Pathology, Universitätsmedizin der Johannes Gutenberg Universität Mainz, Germany.
}

$\triangle$ Corresponding author: Ulrike Dapunt, MD, Center for Orthopaedics, Trauma Surgery and Spinal Cord Injury, Heidelberg University Hospital, Schlierbacher Landstrasse 200a, 69118 Heidelberg, Germany. E-mail: ulrike.dapunt@med.uni-heidelberg.de; Tel.: +49-6221-56-25000.

() The author(s). This is an open access article distributed under the terms of the Creative Commons Attribution License (https://creativecommons.org/licenses/by/4.0/). See http:/ /ivyspring.com/terms for full terms and conditions.

Received: 2020.07.03; Accepted: 2020.10.07; Published: 2021.01.01

\begin{abstract}
Background: Implant loosening - either infectious or aseptic- is a still a major complication in the field of orthopaedic surgery. In both cases, a pro-inflammatory peri-prosthetic environment is generated by the immune system - either triggered by bacteria or by implant wear particles - which leads to osteoclast differentiation and osteolysis. Since infectious cases in particular often require multiple revision surgeries, we wondered whether commonly used surgical suture material may also activate the immune system and thus contribute to loss of bone substance by generation of osteoclasts.

Methods: Tissue samples from patients suffering from infectious implant loosening were collected intraoperatively and presence of osteoclasts was evaluated by histopathology and immunohistochemistry. Further on, human monocytes were isolated from peripheral blood and stimulated with surgical suture material. Cell supernatant samples were collected and ELISA analysis for the pro-inflammatory cytokine IL-8 was performed. These experiments were additionally carried out on ivory slices to demonstrate functionality of osteoclasts. Whole blood samples were incubated with surgical suture material and up-regulation of activation-associated cell surface markers $\mathrm{CDIlb}$ and $\mathrm{CD} 66 \mathrm{~b}$ on neutrophils was evaluated by flow cytofluorometry analysis.

Results: We were able to demonstrate that multinucleated giant cells form in direct vicinity to surgical suture material. These cells stained positive for cathepsin $\mathrm{K}$, which is a typical protease found in osteoclasts. By in vitro analysis, we were able to show that monocytes differentiated into osteoclasts when stimulated with surgical suture material. Resorption pits on ivory slices provided proof that the osteoclasts were functional. Release of IL-8 into cell supernatant was increased after stimulation with suture material and was further enhanced if minor amounts of bacterial lipoteichoic acid (LTA) were added. Neutrophils were also activated by surgical suture material and up-regulation of CDIlb and CD66b could be seen.

Conclusion: We were able to demonstrate that surgical suture material induces a pro-inflammatory response of immune cells which leads to osteoclast differentiation, in particular in combination with bacterial infection. In conclusion, surgical suture material -aside from bacteria and implant wear particles- is a contributing factor in implant loosening.
\end{abstract}

Key words: Osteoclast, osteolysis, surgical suture material, implant-associated infection, Interleukin-8

\section{Introduction}

Even though total joint replacement of the hip and knee is considered one of the most successful surgical procedures in the field of orthopaedics, infectious or aseptic implant loosening is still a severe complication [1-4].
In the case of infections, bacteria form biofilm colonies on an implant surface, which makes them more difficult to detect and to treat. Patients often suffer from a prolonged treatment course, which usually requires extensive antibiotic treatment and 
multiple revision surgeries; possibly including implant-exchange due to loosening [5-7].

In our previous work, we were able to demonstrate that the pro-inflammatory periprosthetic microenvironment, which is generated by a persistent immune response, leads to increased osteoclast generation, bone degradation and in consequence implant loosening [8-10].

We were able to characterize the cellular infiltrate and identify various pro-inflammatory cytokines that are enhanced in these cases and that stimulate osteoclast generation [11].

In the case of so-called aseptic implant loosening where no bacteria are detected, osteolysis is presumably induced by an inflammatory reaction towards implant wear particles [4]. We also evaluated aseptic cases in our previous studies and were able to show, that the pro-inflammatory peri-prosthetic environment is very similar to that found in infection $[11,12]$. However, the response was less pronounced for the relevant cytokines. This finding corresponds with the clinical observation that patients with aseptic loosening usually become symptomatic later on compared to infectious cases, but the inflammatory response will ultimately also lead to generation of osteoclasts and hence implant loosening.

On the other hand, because biofilm infections are difficult to detect and up to $40 \%$ of falsely - negative cases have been reported, the question whether aseptic loosening is truly "aseptic" remains a subject of much debate [13-15]. Moreover, it has been shown that endotoxin adherent on orthopaedic wear particles induces significantly higher cytokine production and osteoclast differentiation, indicative of a combination of factors that eventually trigger an enhanced and persistent pro-inflammatory immune response [16, 17].

In this context, we were interested whether surgical suture material - a foreign body material omnipresent in surgical sites - might similarly contribute to osteoclast generation and further on implant loosening. Several reports can be found in literature that support the notion of an inflammatory response directed towards surgical suture material [18-21]. Multinucleated giant cells have been detected in direct vicinity to suture material via histopathology [22]. Lock and al co-cultivated human monocyte THP-1 cells with seven commonly used suture materials and were able to demonstrate by gene expression and protein secretion analyses that certain sutures in particular induced up-regulation of pro-inflammatory cytokines [23].

Aim of this study was to investigate whether multinucleated giant cells in association with surgical suture material are in fact functional osteoclasts, capable of degrading bone substance and therefore also contributing to implant loosening.

\section{Materials and Methods}

\section{Patients}

Patients $(n=5)$ who underwent revision surgery due to a prosthetic infection with loosening of the implant were included in the study. Diagnosis of loosening of the implant was based on patients' complaints, clinical examination, and examination by conventional X-ray and/or CT scan.

\section{Collection of tissue samples and histology}

From five patients with an infection tissue samples were taken from sites of osteolysis. The study was approved by the local ethics committee of Heidelberg University (No. 206/2005).

The tissue specimens were formalin-fixed, decalcified in ethylenediaminetetraacetic acid (EDTA), and paraffin embedded. Haematoxylin and eosin (H\&E) staining was performed. The biopsies were examined, the diagnosis of an acute or chronic osteomyelitis was made, and the cellular infiltrates in particular the osteoclasts were evaluated. The paraffin-embedded tissue sections $(3-4 \mu \mathrm{m})$ were also used for immunohistochemical analyses. Immunostaining was performed as previously described using the avidin-biotin complex method [24]. Prior to antibody incubation, heat pretreatment in citrate buffer (pH 6.1) was performed. As primary antibody, the monoclonal mouse anti cathepsin K (Calbiochem, San Diego, USA) was used.

\section{Isolation of monocytes}

Monocytes were isolated from the peripheral blood of healthy donors (informed consent was obtained and the institutional guidelines were observed). The blood was layered on Polymorphprep (Axis Shield, Oslo, Norway), and the monocyte fraction was recovered. Monocytes were seeded into 24-well dishes (NuncTM, Wiesbaden, Germany) at a concentration of $3 \times 10^{6}$ per well and selected by adherence after incubation for 2 hours in RPMI containing $1 \%$ glutamine, and $1 \%$ penicillin/ streptomycin (medium and supplements were obtained from Gibco Life Technologies, Darmstadt, Germany). Monocytes were carefully washed to remove any non-adherent cells and $1 \mathrm{~mL}$ of RPMI containing $10 \%$ fetal calf serum, $1 \%$ glutamine, and $1 \%$ penicillin/streptomycin (medium and supplements were obtained from Gibco Life Technologies, Darmstadt, Germany) was added to each well. 


\section{ELISA}

IL-8 level in cell culture supernatants were determined using commercially available ELISA kits according to the protocol provided by the manufacturer (R\&D Systems, Minneapolis, USA).

\section{Surgical suture material}

Coated VICRYL (polyglactin 910) was used for experiments, because this type of suture material was used for deep fascial and subcutaneous layers in patients. Sutures $(0,270 \mathrm{~g})$ were shredded using a scalpel and resuspended in $20 \mathrm{~mL}$ RPMI containing $10 \%$ fetal calf serum and $1 \%$ penicillin/streptomycin.

\section{Generation of osteoclasts}

Monocytes were prepared as described above. For stimulation, the following was added: -medium only, -LTA 0,1 $\mu \mathrm{g} / \mathrm{mL}$ (Sigma, Munich, Germany), -surgical suture material $300 \mu \mathrm{g} / \mathrm{ml}$, LTA $0,1 \mu \mathrm{g} / \mathrm{ml}$ and surgical suture material $300 \mu \mathrm{g} / \mathrm{mL}$, - M-CSF $25 \mathrm{ng} / \mathrm{mL}$ (R\&D Systems, Minneapolis, USA) and RANKL 50 ng/mL (PeproTech, Hamburg, Germany) (positive control).

Cultures were incubated at $37^{\circ} \mathrm{C}$ in $5 \% \mathrm{CO}_{2}$ for 16 days with a change of medium and removal of nonadherent cells at day 7 and day 14. Cell supernatant samples for IL-8 ELISA were obtained after 48 hours, 7 days and 14 days.

For the follow up differentiation, the cells were fixed with $4 \%$ PFA for 15 minutes at $37^{\circ} \mathrm{C}$ and identified by their morphological appearance as giant cells with multiple nuclei. Binding of FITC-labeled Phalloidin (Sigma-Aldrich; 1:20 dilution for 40 minutes) revealed the typical actin ring formation. Nuclei were stained using DAPI (Invitrogen, Oregon, USA), (diluted $1: 20000$ for 5 minutes).

Fluorescence was visualized using a Digital Fluorescence Microscope (Keyence, Neu-Isenburg, Germany).

Functionality of osteoclasts was tested by differentiating monocytes on ivory slices for 16 days, followed by toluidine blue staining and quantification of the resorption pits by light microscopy (ivory was obtained from the Bundesamt für Umweltschutz, Bonn, Germany).

\section{Functional assay with neutrophils}

To heparinized blood, LTA $0,1 \mu \mathrm{g}, 200 \mu \mathrm{l}$ of suture material suspension and LTA combined with suture material was added, followed by incubation for $30 \mathrm{~min}$. CD11b and CD66b expression was measured by cytofluorometry. Directly labelled antibody to CD11b (FITC) or CD66b (FITC) (both obtained from Beckman Coulter, Krefeld, Germany) were used and for comparison isotypic mouse IgG -
FITC (BD Bioscience Pharmingen, San Jose, CA, USA). The samples were analysed using FACSCalibur (Becton Dickinson, Heidelberg, Germany) and Cell Quest Pro software (Version 4.0.2, Becton Dickinson).

\section{Statistical Analysis}

Differences between groups were calculated using Mann-Whitney test and unpaired t-test using Graph Pad Prism 8.4.1 software. Significance level was determined as $p<0.05$.

\section{Results}

Tissue samples were collected intraoperatively from areas of osteolysis from patients suffering from infectious implant loosening (Figure 1). Patients' clinical data are shown in Table 1. Diagnosis of implant-associated infection was confirmed by histology (>23 neutrophils per 10 high power fields) [25]. H\&E staining showed multinucleated giant cells in direct vicinity to surgical suture material. Additionally, immunohistochemical staining for cathepsin $\mathrm{K}$, which is characteristic for osteoclasts, was performed and giant cells in association with suture material showed positive results (Figure 2A-D).

Table 1. Patients' clinical data. Five patients with infectious implant loosening who underwent removal of the implant were included in the study. Microbiological evaluation of tissue samples revealed multiple bacteria species in one patient

\begin{tabular}{|c|c|}
\hline & \\
\hline \multicolumn{2}{|l|}{ Gender } \\
\hline Male & $\mathrm{n}=3$ \\
\hline Female & $\mathrm{n}=2$ \\
\hline \multicolumn{2}{|l|}{ Age (years) } \\
\hline Mean ( \pm standard deviation) & $68( \pm 18.96)$ \\
\hline Median (range) & $72(41-91)$ \\
\hline \multicolumn{2}{|c|}{ Loosening of total joint replacement } \\
\hline Hip & $\mathrm{n}=1$ \\
\hline Knee & $\mathrm{n}=4$ \\
\hline \multicolumn{2}{|c|}{ CRP (C-reactive protein) concentration } \\
\hline Mean ( \pm standard deviation) & $116.9( \pm 113.7) \mathrm{mg} / \mathrm{L}$ \\
\hline \multicolumn{2}{|l|}{ WBC (white blood cell count) } \\
\hline Mean ( \pm standard deviation) & $8.4( \pm 1.4) / \mathrm{nl}$ \\
\hline \multicolumn{2}{|c|}{ Bacteria species detected by culture of tissue samples } \\
\hline Enterococcus faecalis & $\mathrm{n}=1$ \\
\hline Staphylococcus aureus & $\mathrm{n}=3$ \\
\hline Coagulase-negative Staphylococci & $\mathrm{n}=2$ \\
\hline
\end{tabular}

These results were followed up by in vitro analysis. Monocytes were isolated and stimulated with surgical suture material and/or LTA (lipoteichoic acid), the latter a constituent of the cell wall of gram-positive bacteria.

Samples of cell supernatant were collected after 48 hours, 7 days and 14 days for IL-8 ELISA analysis (Table 2; Figure 3). Experiments were repeated twice and the results after 7 days of incubation are shown. 


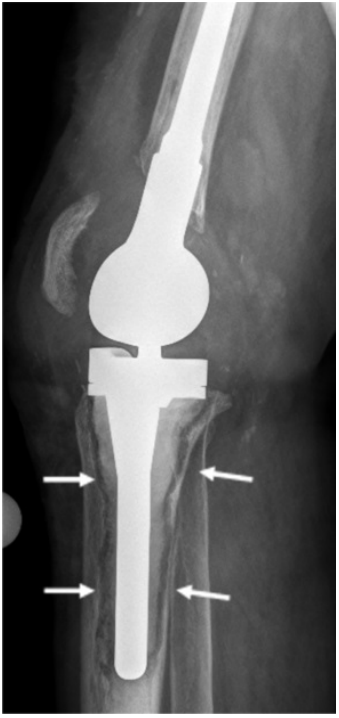

Figure 1. X-ray of a patient suffering from infectious implant loosening of a total knee replacement. Radiolucent lines can be seen particularly around the tibial implant component, indicative of loosening (white arrows).

After 7 days of incubation with surgical suture material, IL-8 release was significantly higher compared to the control sample ( $p=0.0127$ as tested by unpaired $t$-test).

The individual response varied greatly between different donors and therefore statistical significance was not reached when comparing LTA and LTA+suture. However, an increased response to LTA+suture could be observed for each donor as shown in Figure 3, which indicates that a combination of activating substances can further enhance the pro-inflammatory response.

The same experiments were also carried out on ivory slices and essentially showed similar results. However, the overall expression of IL-8 was much higher, which could be due to different conditions on ivory slices or 6-well dishes instead of 24-well dishes, for example. That is why these experiments were not combined in statistical calculations. Nevertheless, the relative increase of IL- 8 was the same under both experimental settings.

Further on, cells were evaluated by fluorescence microscopy. Giant cells attached to surgical suture material and showed multiple nuclei, as well as actin rings, known as typical features of osteoclasts (Figure 4A,B).

In order to evaluate functionality of these cells, similar experiments were performed on ivory slices and resorption pits were evaluated by two independent observers.

As shown in Table 3, more resorption pits were found after stimulation with LTA and with surgical suture material. The most resorption pits were seen after stimulation with LTA and suture material together, which again underlines the notion that a combination of stimuli can further enhance the inflammatory response (Figure 5).
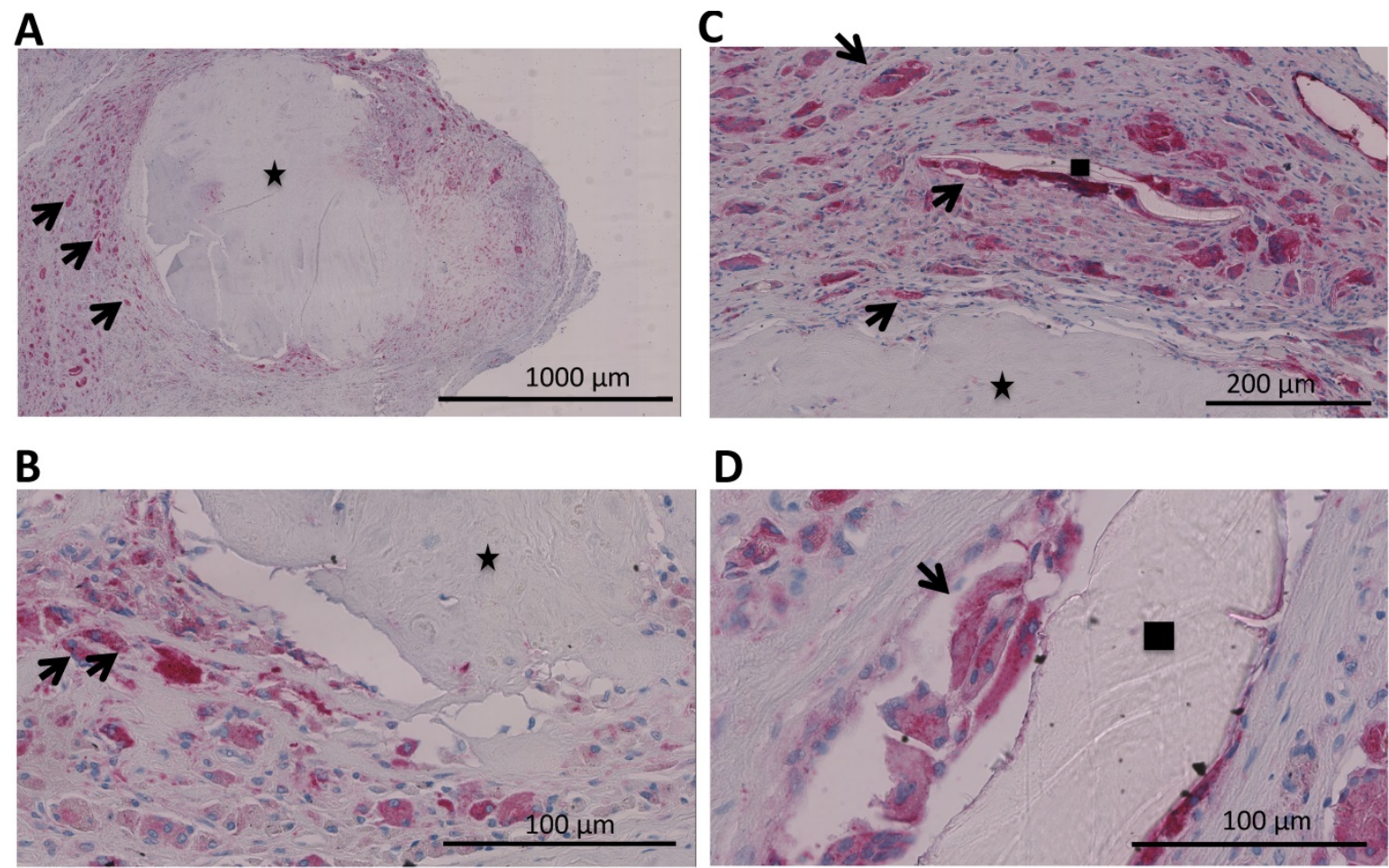

D

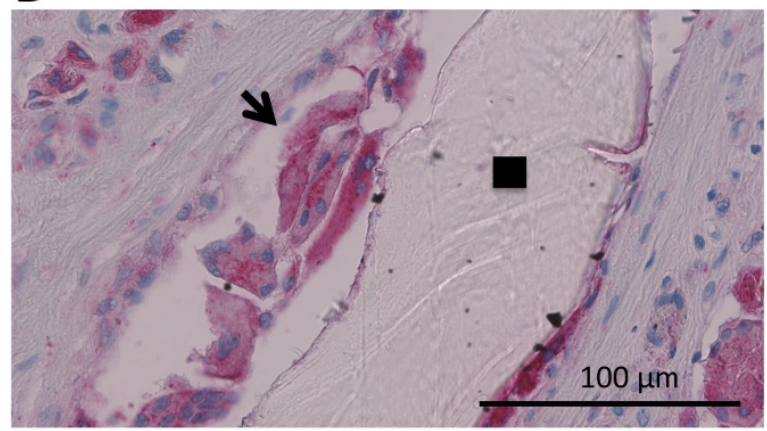

Figure 2. A-D. Analysis of a patients' biopsy with prosthetic joint infection. A,B Eroded bone (identified by black asterix) is seen and next to it osteoclasts (identified by an antibody for cathepsin K (dark red) and multiple nuclei (blue)) (black arrows). C,D Multinucleated giant cells that were positive for cathepsin K (osteoclasts; black arrows) could also be found in direct vicinity to surgical suture material (identified by black square). 
Table 2. IL-8 (pg/ml) measured in cell supernatant of monocytes after 48 hours, 7 days and 14 days of incubation. Differences between medium (control) and groups were calculated using unpaired $t$-test with $p<0.05$. Experiments were repeated twice and additionally also on ivory slices

\begin{tabular}{llllll}
\hline & $\begin{array}{l}\text { Medium IL-8 } \\
(\mathrm{pg} / \mathrm{ml})\end{array}$ & $\begin{array}{l}\text { LTA IL-8 } \\
(\mathrm{pg} / \mathrm{ml})\end{array}$ & $\begin{array}{l}\text { Surgical suture material IL-8 } \\
(\mathrm{pg} / \mathrm{ml})\end{array}$ & $\begin{array}{l}\text { LTA+surgical suture material IL-8 } \\
(\mathrm{pg} / \mathrm{ml})\end{array}$ & $\begin{array}{l}\text { RANKL+M-CSF (positive control) } \\
\mathrm{IL}-8(\mathrm{pg} / \mathrm{ml})\end{array}$ \\
\hline $48 \mathrm{~h}$ & 61,7 & 497,3 & 101,6 & 890 & 242,4 \\
$48 \mathrm{~h}$ & 144,3 & 2224,8 & 127 & 1793,9 & 550,4 \\
$7 \mathrm{~d}$ & 175,5 & $1319,5^{*}(p=0.0393)$ & $369,6^{*}(p=0.0127)$ & 4688 & $974,6^{*}(p=0.0156)$ \\
$7 \mathrm{~d}$ & 129,7 & $1916,2^{*}$ & $355,7^{*}$ & 2362,8 & $795,2^{*}$ \\
$14 \mathrm{~d}$ & 98,6 & 281,6 & 237,8 & 483,9 & 217,3 \\
$14 \mathrm{~d}$ & 72,1 & 2312,3 & 80,4 & 3191,5 & 186,2 \\
\hline
\end{tabular}

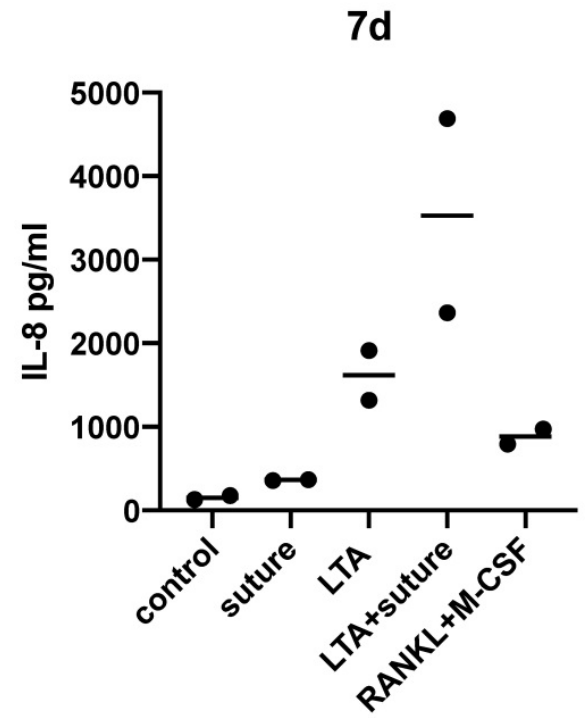

Figure 3. Cell supernatants were collected after 7 days and IL-8 ELISA (pg/ml) was performed. Increased IL-8 release could be detected compared to medium only (control).

Table 3. Resorption pits were evaluated by two independent observers. Stimulation with LTA and surgical suture material in particular showed the highest numbers of resorption pits. Experiments were carried out multiple times. Results of one experiment are shown

\begin{tabular}{lll}
\hline & $\begin{array}{l}\text { Number of resorption } \\
\text { pits, observer 1 }\end{array}$ & $\begin{array}{l}\text { Number of resorption } \\
\text { pits, observer 2 }\end{array}$ \\
\hline $\begin{array}{l}\text { Medium (control) } \\
\text { LTA }\end{array}$ & $\mathrm{n}=0$ & $\mathrm{n}=0$ \\
Surgical suture material & $\mathrm{n}=81$ & $\mathrm{n}=70$ \\
$\begin{array}{l}\text { LTA+surgical suture } \\
\text { material }\end{array}$ & $\mathrm{n}=201$ & $\mathrm{n}=110$ \\
$\begin{array}{l}\text { RANKL/M-CSF } \\
\text { (positive control) }\end{array}$ & $\mathrm{n}=59$ & $\mathrm{n}=166$
\end{tabular}

In our previous work we were able to demonstrate that polymorphonuclear neutrophils (PMN) are predominant in implant-associated infections and that these cells release proinflammatory cytokines, such as MRP-14 and IL-8, which leads to osteoclast generation. Therefore, we were interested in evaluating whether PMNs were also affected by surgical suture material. After stimulating whole blood samples with suture material and LTA, we were able to demonstrate an increase of the activation-associated cell surface markers CD11b and CD66b on neutrophils (Figure 6A-C). Although each donor showed an enhanced response after stimulation with surgical suture material, the individual response varied greatly between different donors, and therefore statistical significance was not reached (Figure 6C).

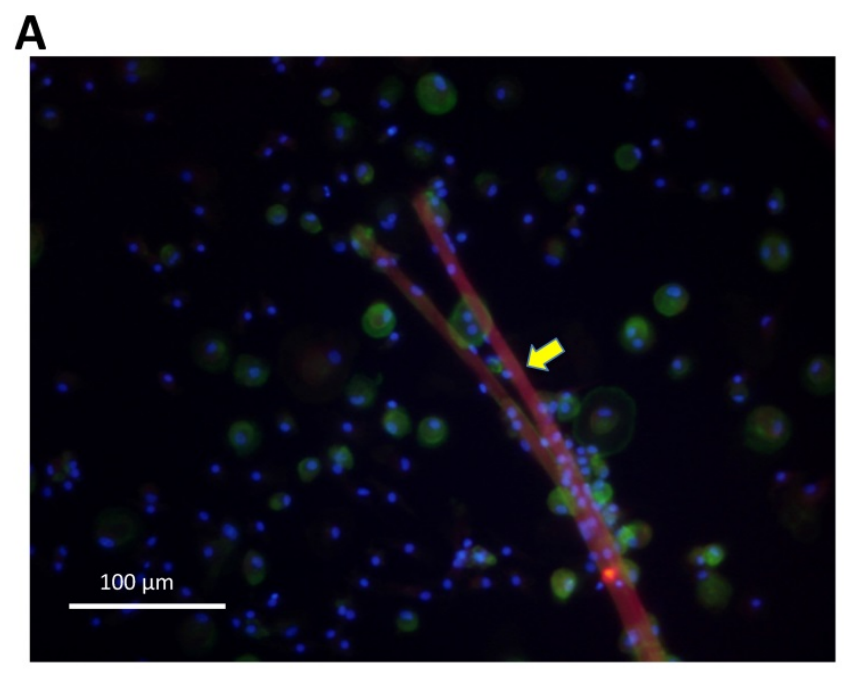

B

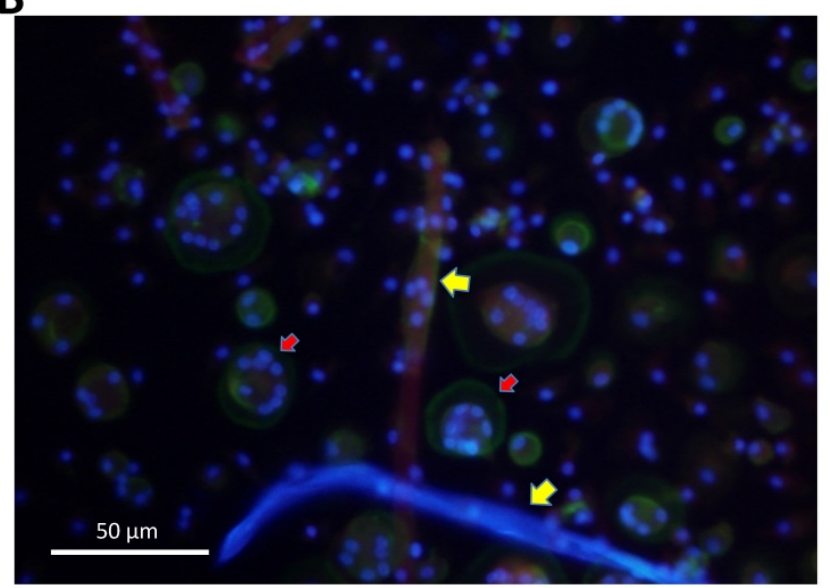

Figure 4. A. Monocytes were isolated from peripheral blood and stimulated with surgical suture material (red). After 16 days multinucleated giant cells had formed in direct vicinity to surgical suture material (yellow arrows), as demonstrated by fluorescence microscopy. B. Cells that were in direct vicinity to surgical suture material (yellow arrows) showed multiple nuclei (blue) and actin rings (geen colour; indicated by red arrows), which are typical for osteoclasts as demonstrated by fluorescence microscopy. 


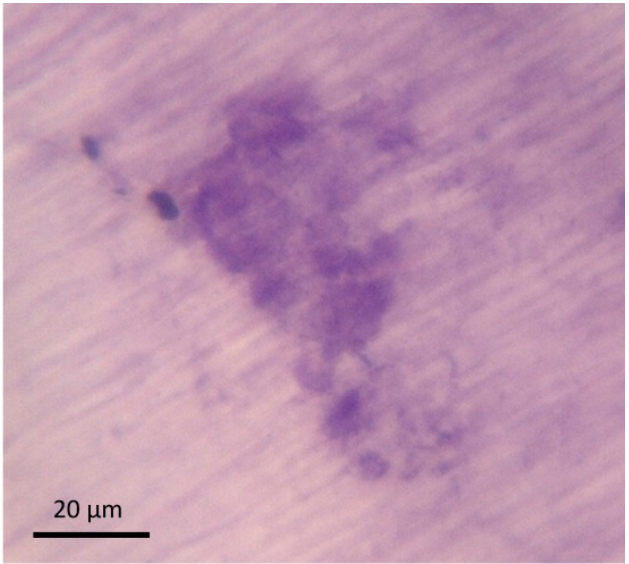

Figure 5. After incubation for 16 days, ivory slices were stained with toluidine blue and resorption pits were counted by two independent observers.

\section{Discussion}

In our previous work, we were interested in evaluating the pro-inflammatory environment in implant-associated infections and aseptic implant loosening, particularly with regard to bone resorption. Similar local defence mechanisms are activated, although the number and composition of the cellular infiltrate vary [11]. In both cases, phagocytosis of either bacteria or of wear particles is initiated by monocytic cells and in implant-associated infections also by neutrophils. Phagocytosis is associated with a release of various cytokines which results in the generation of a pro-inflammatory environment. As the inflammatory response progresses, monocytes differentiate into osteoclasts, the latter being the only cell type capable of degrading bone. Because the initial insult persists (phagocytosis of bacteria or wear particles), so does the inflammatory response, which eventually leads to osteolysis and further on implant loosening $[4,9,10$, $12,26]$.

We evaluated tissue samples that were collected intra-operatively from patients suffering from implant-associated infections. Histopatholgical analysis revealed that multinucleated giant cells were detected in areas of osteolysis. Due to their positivity for the specific protease cathepsin K, they could be identified as osteoclasts.

However, multinuclear giant cells were not merely distributed along bone substance. We also observed a conspicuous number of giant cells in direct vicinity to surgical suture material. The fact that a "foreign body", such as calcifications, wear particles, uremic cristals, or indeed surgical suture material can induce a monocytic immune reaction with generation of foreign body macrophages, has been known, however whether such type of immune reaction can also lead to osteoclastogenesis was still unknown[4,
21]. Therefore, we were interested whether these cells are in fact also functional osteoclasts.
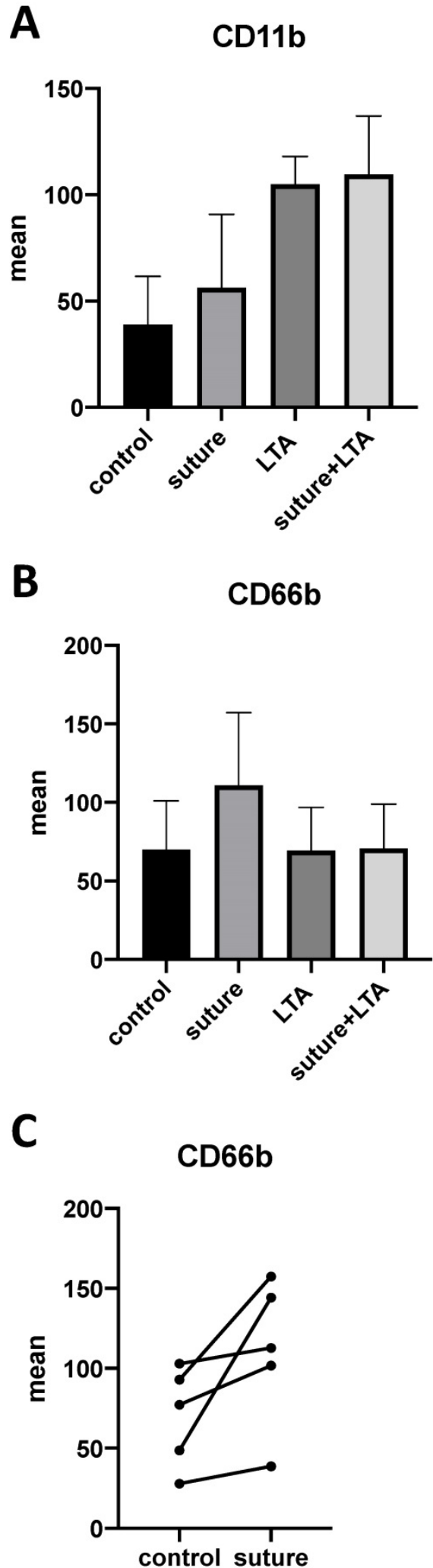

Figure 6. A-C. Whole blood samples were stimulated with surgical suture material and/or LTA. A,B. Up-regulation of the activation-associated cell surface markers CD11b and CD66b could be observed by FACS (fluorescence-activated cell sorting) analysis. C. Individual response among different donors varied greatly, but an up-regulation could be seen for each donor. The data for 5 individuals are shown. 
The formation of multinuclear giant cells in response to surgical suture material has been described in literature. Lovric et al. used a murine synovial airpouch model to demonstrate formation of multinuclear giant cells around various suture materials, as well as an enhanced expression of matrix metalloproteinases (MMP) -1,-2,-9, and -13 in particular when suture wear particles were compared to intact sutures [18]. Lambertz et al. evaluated the response to 6 different surgical suture materials by histopathology in a rat model. They described a foreign body granuloma and a comet tail-like infiltrate (CTI) for each suture material, however the CTI was particularly enhanced when Vicryl (polyglactin 910) was used [22].

An in vitro study by Lock et al. using human monocyte THP-1 cells showed similar results concerning Vicryl sutures. Enhanced gene expression of the pro-inflammatory cytokine IL-8, as well as a decrease of the anti-inflammatory cytokine TGF $\beta 1$ was observed [23].

Moreover, there are also clinical reports available, which describe that revision surgeries were performed due to suspected post-operative infections, but histopathological evaluation of tissue samples revealed granulomatous inflammation due to suture material (Vicryl) instead [27, 28].

Vicryl is an absorbable suture which is composed of $90 \%$ glycolide and 10\% L-lactide and is widely used for closure of deep fascial and subcutaneous layers [29].

In this study, we also used Vicryl polyglactin 910 sutures for our in vitro experiments, because this type of surgical suture material was used in all patients.

In order to prove that multinucleated giant cells in direct vicinity to surgical suture material were also functional osteoclasts, immunohistochemical staining for cathepsin $\mathrm{K}$ was performed. Cathepsin $\mathrm{K}$ is a protease which is involved in bone resorption and has been described as typical for osteoclasts [30-32].

As shown in Figure 2, multinucleated giant cells were indeed positive for cathepsin $\mathrm{K}$ and therefore follow-up in vitro experiments were performed.

We were able to show by fluorescence microscopy that human monocytes differentiated into osteoclasts when stimulated with surgical suture material. Cells showed multiple nuclei, as well as the osteoclast-typical actin ring. Functionality of osteoclasts was tested by performing the experiments on ivory slices. Resorption pits were evaluated and higher numbers were counted after stimulation with surgical suture material. Furthermore, cell supernatant samples were collected and increased release of IL-8 was observed. Interleukin- 8 is a wellestablished pro-inflammatory cytokine and has been previously linked to osteoclast generation. Similarly, Lock et al. also demonstrated in their study that IL-8 gene expression in monocyte THP-1 cells was enhanced following stimulation with Vicryl sutures [23].

Because the cellular infiltrate in implantassociated infections comprises not merely macrophages but also high numbers of neutrophils, we were interested in the effect of surgical suture material on these cells. Our results show that the activationassociated cell surface markers CD11b and CD66b were up-regulated after stimulation with surgical suture material, suggesting that not only macrophages but also neutrophils recognize and respond to suture material. As previously reported in literature, the infiltration of neutrophils in chronic inflammatory diseases correlates with the number of osteoclasts found in tissue samples [33]. Activated neutrophils might also directly stimulate osteoclastogenesis via release of IL-8, therefore linking inflammation and bone erosion.

Additionally, we were interested in evaluating whether a combination of different stimuli could further enhance the pro-inflammatory response. Therefore, minor amounts of LTA -which is a major component of the bacterial cell wall of gram-positive bacteria-, to mimic infection, was added. We were able to show that the numbers of resorption pits as well as the release of IL-8 were further increased when human monocytes were stimulated with minor amounts of LTA and surgical suture material together.

The additional effect that minor amounts of bacterial products or endotoxins might have on immune cells has been well-described in particular in regard to aseptic implant-loosening. Bi et al. were able to show in vitro and in vivo that adherent endotoxin on titanium wear particles enhanced proinflammatory cytokine production and osteoclast differentiation [17] and their in vivo experiments demonstrated that particle-induced osteolysis was reduced by $50-70 \%$ if adherent endotoxin was removed.

Our experiments indicate that bacterial components and surgical suture materials might also elicit a synergistic effect on immune cells, which leads to increased osteoclast generation.

These findings might not only be relevant in the context of orthopaedic implant-associated infections. It has been previously described in literature that macrophages release a variety of products including proteases after induction by phagocytosis and/or inflammatory stimuli. The release might be enhanced, particularly if phagocytosis of large particles is unsuccessful and has been described to contribute to 
tissue damage in chronic inflammatory disease such as rheumatoid arthritis [34, 35].

Impaired phagocytosis by macrophages might also have effects on tissue repair processes and could result in severe post-surgical complications such as anastomotic leakage [36].

\section{Conclusions}

Implant-associated infections are difficult to treat and often require multiple revision surgeries due to persistent infection. Our results show that in these cases, the immune system is constantly activated not only by bacterial components but also by surgical suture material which is renewed during every revision surgery. We were able to demonstrate for the first time, that the generation of functional osteoclasts can also be induced by surgical suture material and is aggravated under septic conditions, which further contributes to implant-loosening. This is a further step to understand implant loosing after joint replacement.

\section{Acknowledgements}

\section{Author Contributions}

All authors have made substantial contributions to conception and design, acquisition of data, analysis and interpretation of data and have been involved in drafting the manuscript or revising it critically for important intellectual content.

All authors concur with the submission of the article, have given final approval of the version to be published and agree to be accountable for all aspects of the work in ensuring that questions related to the accuracy or integrity of any part of the work are appropriately investigated and resolved.

\section{Competing Interests}

The authors have declared that no competing interest exists.

\section{References}

1. Learmonth ID, Young C, Rorabeck C. The operation of the century: total hip replacement. Lancet. 2007; 370: 1508-19.

2. Haenle M, Skripitz C, Mittelmeier W, Skripitz R. [Economic impact of infected total hip arthroplasty in the German diagnosis-related groups system]. Orthopade. 2012; 41: 467-76.

3. Zimmerli W, Trampuz A, Ochsner PE. Prosthetic-joint infections. N Engl J Med. 2004; 351: 1645-54.

4. Goodman SB. Wear particles, periprosthetic osteolysis and the immune system. Biomaterials. 2007; 28: 5044-8.

5. Costerton JW, Stewart PS, Greenberg EP. Bacterial biofilms: a common cause of persistent infections. Science. 1999; 284: 1318-22.

6. Montanaro L, Speziale P, Campoccia D, Ravaioli S, Cangini I, Pietrocola G, et al.. Scenery of Staphylococcus implant infections in orthopedics. Future Microbiol. 2011; 6: 1329-49.

7. Stoodley P, Ehrlich GD, Sedghizadeh PP, Hall-Stoodley L, Baratz ME, Altman DT, et al.. Orthopaedic biofilm infections. Curr Orthop Pract. 2011; 22: 558-63.

8. Dapunt U, Giese T, Stegmaier S, Moghaddam A, Hansch GM. The osteoblast as an inflammatory cell: production of cytokines in response to bacteria and components of bacterial biofilms. BMC Musculoskelet Disord. 2016; 17: 243.

9. Dapunt U, Maurer S, Giese T, Gaida MM, Hansch GM. The macrophage inflammatory proteins MIP1alpha (CCL3) and MIP2alpha (CXCL2) in implant-associated osteomyelitis: linking inflammation to bone degradation. Mediators Inflamm. 2014; 2014: 728619.

10. Dapunt U, Giese T, Maurer S, Stegmaier S, Prior B, Hansch GM, et al.. Neutrophil-derived MRP-14 is up-regulated in infectious osteomyelitis and stimulates osteoclast generation. J Leukoc Biol. 2015.

11. Dapunt U, Giese T, Lasitschka F, Lehner B, Ewerbeck V, Hansch GM. Osteoclast Generation and Cytokine Profile at Prosthetic Interfaces: A Study on Tissue of Patients with Aseptic Loosening or Implant-Associated Infections. Eur J Inflamm. 2014; 12: 147-59.

12. Dapunt U, Giese T, Lasitschka F, Reinders J, Lehner B, Kretzer JP, et al.. On the inflammatory response in metal-on-metal implants. J Transl Med. 2014; 12 : 74.

13. Berbari EF, Marculescu C, Sia I, Lahr BD, Hanssen AD, Steckelberg JM, et al.. Culture-negative prosthetic joint infection. Clin Infect Dis. 2007; 45: 1113-9.

14. Stefansdottir A, Johansson D, Knutson K, Lidgren L, Robertsson O. Microbiology of the infected knee arthroplasty: report from the Swedish Knee Arthroplasty Register on 426 surgically revised cases. Scand J Infect Dis. 2009; 41: 831-40.

15. Neut D, van Horn JR, van Kooten TG, van der Mei HC, Busscher HJ. Detection of biomaterial-associated infections in orthopaedic joint implants. Clin Orthop Relat Res. 2003; p: 261-8.

16. Nalepka JL, Lee MJ, Kraay MJ, Marcus RE, Goldberg VM, Chen X, et al.. Lipopolysaccharide found in aseptic loosening of patients with inflammatory arthritis. Clin Orthop Relat Res. 2006; 451: 229-35.

17. Bi Y, Seabold JM, Kaar SG, Ragab AA, Goldberg VM, Anderson JM, et al.. Adherent endotoxin on orthopedic wear particles stimulates cytokine production and osteoclast differentiation. J Bone Miner Res. 2001; 16: 2082-91.

18. Lovric V, Goldberg MJ, Heuberer PR, Oliver RA, Stone D, Laky B, et al.. Suture wear particles cause a significant inflammatory response in a murine synovial airpouch model. J Orthop Surg Res. 2018; 13: 311.

19. Kakoei S, Baghaei F, Dabiri S, Parirokh M, Kakooei S. A comparative in vivo study of tissue reactions to four suturing materials. Iran Endod J. 2010; 5: 69-73.

20. Eickhoff RM, Bolle T, Kossel K, Heise D, Kroh A, Lambertz A, et al.. Improved biocompatibility of profiled sutures through lower macrophages adhesion. J Biomed Mater Res B Appl Biomater. 2019; 107: 1772-8.

21. Andrade MG, Weissman R, Reis SR. Tissue reaction and surface morphology of absorbable sutures after in vivo exposure. J Mater Sci Mater Med. 2006; 17: 949-61.

22. Lambertz A, Schroder KM, Schob DS, Binnebosel M, Anurov M, Klinge U, et al.. Polyvinylidene Fluoride as a Suture Material: Evaluation of Comet Tail-Like Infiltrate and Foreign Body Granuloma. Eur Surg Res. 2015; 55: 1-11.

23. Lock AM, Gao R, Naot D, Coleman B, Cornish J, Musson DS. Induction of immune gene expression and inflammatory mediator release by commonly used surgical suture materials: an experimental in vitro study. Patient Saf Surg. 2017; 11: 16.

24. Gaida MM MB, Stegmaier S, Schirmacher P, Wagner C, Hänsch GM. Polymorphonuclear neutrophils in osteomyelitis: link to osteoclast generation and bone resorption. Europ J of Inflamm. 2012.

25. Morawietz L, Classen RA, Schroder JH, Dynybil C, Perka C, Skwara A, et al.. Proposal for a histopathological consensus classification of the periprosthetic interface membrane. J Clin Pathol. 2006; 59: 591-7.

26. Hänsch G. Host Defence against Bacterial Biofilms: "Mission Impossible"? ISRN Immunology. 2012; 2012: 17.

27. Pierannunzii L, Fossali A, De Lucia O, Guarino A. Suture-related pseudoinfection after total hip arthroplasty. J Orthop Traumatol. 2015; 16: 59-65.

28. Sayegh S, Bernard L, Stern R, Pache JC, Szalay I, Hoffmeyer P. Suture granuloma mimicking infection following total hip arthroplasty. A report of three cases. J Bone Joint Surg Am. 2003; 85: 2006-9.

29. Tajirian AL, Goldberg DJ. A review of sutures and other skin closure materials. J Cosmet Laser Ther. 2010; 12: 296-302.

30. Bossard MJ, Tomaszek TA, Thompson SK, Amegadzie BY, Hanning CR, Jones $\mathrm{C}$, et al.. Proteolytic activity of human osteoclast cathepsin K. Expression, purification, activation, and substrate identification. J Biol Chem. 1996; 271: 12517-24.

31. Fujisaki K, Tanabe N, Suzuki N, Kawato T, Takeichi O, Tsuzukibashi O, et al.. Receptor activator of NF-kappaB ligand induces the expression of carbonic anhydrase II, cathepsin $\mathrm{K}$, and matrix metalloproteinase-9 in osteoclast precursor RAW264.7 cells. Life Sci. 2007; 80: 1311-8. 
32. Wilson SR, Peters C, Saftig P, Bromme D. Cathepsin K activity-dependent regulation of osteoclast actin ring formation and bone resorption. J Biol Chem. 2009; 284: 2584-92.

33. Gaida MM MB, Stegmaier S, Schirmacher P, Wagner C, Hänsch GM. Polymorphonuclear neutrophils in osteomyelitis: link to osteoclast generation and bone resorption. Eur J Inflamm. 2012.

34. Gordon S, Newman W, Bloom B. Macrophage proteases and rheumatic diseases: regulation of plasminogen activator by thymus-derived lymphocytes. Agents Actions. 1978; 8: 19-26.

35. Davies P, Allison AC. The macrophage as a secretory cell in chronic inflammation. Agents Actions. 1976; 6: 60-74.

36. Shi J, Wu Z, Li Z, Ji J. Roles of Macrophage Subtypes in Bowel Anastomotic Healing and Anastomotic Leakage. J Immunol Res. 2018; 2018: 6827237. 